\title{
Predictors of survival and good neurological outcomes after in-hospital cardiac arrest
}

\author{
Min Jee Lee ${ }^{1}$, Ji Ho Ryu ${ }^{1, *}$, Mun Ki Min ${ }^{1}$, Dae Sup Lee ${ }^{1}$, Seok Ran Yeom², \\ Byung kwan Bae${ }^{2}$, Young Mo Cho' ${ }^{2}$, Soon Chang Park ${ }^{2}$
}

\author{
${ }^{1}$ Department of Emergency Medicine, \\ Pusan National University Yangsan \\ Hospital, College of Medicine, Pusan \\ National University, Yangsan, Korea \\ ${ }^{2}$ Department of Emergency Medicine, \\ Pusan National University Hospital, \\ College of Medicine, Pusan National \\ University, Busan, Korea \\ *Correspondence \\ pnuem@pusan.ac.kr \\ (Ji Ho Ryu)
}

\begin{abstract}
Objectives: This study aimed to investigate the effect of the code blue activation system and factors affecting patients' survival to discharge and neurologic outcomes after inhospital cardiac arrest.

Methods: We retrospectively reviewed the data of patients aged $\geq 18$ years who experienced in-hospital cardiac arrest between July 2014 and September 2019 at a tertiary hospital. The outcomes included survival to hospital discharge and neurologic outcomes (cerebral performance category score).

Results: In total, 605 patients were included. The rate of survival to discharge was $21.8 \%$ $(n=132)$, and the rate of sustained return of spontaneous circulation was $69.7 \%(n=422)$. Predisposing conditions, such as sepsis, cancer, pneumonia, and use of vasopressors, were associated with poor prognosis, and the survival rate was low $(P=0.01)$. The rate of survival to discharge was higher in patients who underwent defibrillation (odds ratio: 2.48 , 95\% confidence interval: $1.36-4.53$ ) than in those who did not. The median cardiopulmonary resuscitation (CPR) duration time was 11.0 and $26.5 \mathrm{~min}$ in the survival and non-survival groups, respectively $(P<0.01)$. Code blue activation to CPR team arrival time (advanced cardiovascular life support activation time) was not significantly different within 1 minute in both groups $(P=0.95)$. Similarly, no differences in basic life support activation time and first time to defibrillation were observed between the survival and non-survival groups. Among survivors, factors affecting favorable neurologic outcomes were young age, cerebral performance before CPR, whether witnessed, admission days, and CPR duration.

Conclusions: The compulsory availability of a systematic code blue activation is not sufficient. Further, appropriate monitoring and continuous observation are crucial for improving survival to discharge and neurologic outcomes and preventing cardiac arrest in high-risk patients.
\end{abstract}

\section{Keywords}

Cardiac arrest; Cardiopulmonary resuscitation; Survival; Code blue; Cardiac life support

\section{Introduction}

The American College of Cardiology/American Heart Association (ACC/AHA) 2020 revised guidelines separated the chain of survival for in-hospital cardiac arrest (IHCA) and out-of-hospital cardiac arrest (OHCA) [1]. This separation was necessary because the circumstances surrounding IHCA and OHCA are extremely different [2]. OHCA survival depends on early recognition of the event, early initiation of high-quality cardiopulmonary resuscitation (CPR) by the bystander, appropriate early defibrillation, effective advanced cardiovascular resuscitation procedures, such as drug administration and advanced airway maintenance, and integrated postcardiac arrest care [3-5]. For IHCA, the chain of survival is supposedly well established; hence, a high survival rate and good prognosis can be expected. However, survival-todischarge rates and prognosis of IHCA are different from those of OHCA. The survival-to-discharge rate for OHCA and IHCA are estimated to be about $10 \%$ and $12-25 \%$, respectively [69]. Many IHCA cases involve patients suffering from severe underlying diseases with a low resuscitation potential; $80 \%$ of the patients are usually physiologically and clinically unstable $[10,11]$.

In Korea, the Korea Centers for Disease Control and Prevention research has piloted studies to improve the survival rate of patients with acute cardiac arrest since 2008, and there is a report on survival analysis and prognosis after acute cardiac arrest [12]. However, most of the studies are limited to the analysis of OHCA and cardiac arrest at emergency medical center levels [13]. 
TA B L E 1. Comparison with survival to discharge and in-hospital death group

$\begin{array}{lcccc}\text { Patient variables } & \text { Survival to discharge }(\mathrm{n}=132) & \text { In-hospital death }(\mathrm{n}=473) & \text { Total }(\mathrm{n}=605) & P \text { value } \\ \text { Age }(\text { median, IQR) } & 70.5(54.2-80.7) & 66.0(58.0-73.7) & 67(57.0-76.0) & 0.39 \\ \text { Male, } \mathrm{n}(\%) & 77(20.6) & 296(79.4) & 373(61.6) & 0.37 \\ \text { Predisposing factor, n (\%) } & & & & \\ \text { DM } & 51(38.6) & 154(32.6) & 205(33.9) & 0.19 \\ \text { Sepsis } & 13(9.8) & 126(26.6) & 139(23.0) & <0.01^{*} \\ \text { ACS, this admission } & 26(19.7) & 58(12.3) & 84(13.9) & 0.02^{*} \\ \text { ACS, prior admission } & 15(11.4) & 47(9.9) & 62(10.2) & 0.63 \\ \text { Malignancy } & 24(18.2) & 160(33.8) & 184(30.4) & <0.01^{*} \\ \text { Pneumonia } & 17(12.9) & 126(26.6) & 143(23.6) & <0.01^{*} \\ \text { Renal disease } & 38(28.8) & 122(25.8) & 160(26.4) & 0.49 \\ \text { Hepatic disease } & 10(7.6) & 50(10.6) & 60(9.9) & 0.30 \\ \text { Stroke } & 23(19.2) & 68(14.4) & 91(15.0) & 0.38 \\ \text { Causes, n (\%) } & & & 128(21.2) & 197(32.6) \\ \text { Cardiac } & 38(28.8) & 90(19) & 96(15.9) & <0.01^{*} \\ \text { Respiratory } & 47(35.6) & 150(31.7) & 61(10.1) & 85(14.0)\end{array}$

DM, Diabetes mellitus; ACS, Acute coronary syndrome.

At each general tertiary hospital, for the safety and quality management of patients and the evaluation of certification by medical institutions, the medical staff's ability to perform CPR and response systems are monitored. CPR start time after cardiac arrest, time to CPR team activation, communication system in case of cardiac arrest, defibrillator management, CPR survival rate, and cerebral performance categories (CPC) score are reported as performance indicators. However, information sharing and reporting of results at the academic level are completely absent.

Therefore, our study aimed to assess the prognosis of patients by analyzing the CPR team activation time and CPR time after cardiac arrest in IHCA to determine factors affecting survival to discharge, which reflect the neurological prognosis.

\section{Methods}

This study protocol was reviewed and approved by the Institutional Review Board of our hospital (approval no. 05-2020163).

\subsection{Study settings and population}

This was a retrospective study of patients aged $\geq 18$ years who had IHCA in a tertiary medical hospital from July 2014 to September 2019. The study excluded cardiac arrest in children aged $<18$ years, cardiac arrest during surgeries and procedures, patients with written Do Not Resuscitate (DNR) orders, and recurrent cardiac arrest within 24 hours.

The primary and secondary outcomes were survival to discharge rate and CPC score on discharge. CPC scores of 1 and 2 were classified as good neurological status, and CPC scores of 3, 4, and 5 were classified as poor neurological status [14].

Our hospital has a CPR team trained in Korean advanced life support (KALS) or AHA advanced cardiovascular life support (ACLS) course. The CPR team consists of professors of emergency medicine, pediatrics, cardiology, respiratory medicine, thoracic surgery, anesthesiology, and nurses. When a patient experiences cardiac arrest, the code blue activation system is activated, and the team is subsequently notified of the location of the arrest by hospital broadcasting. For CPR quality management, arrest recognition to CPR start time, code blue activation to the arrival time of the CPR team, defibrillator management, return of spontaneous circulation (ROSC), survival-to-discharge rate, and CPC score, among others, are reported in real-time and reviewed by the CPR committee.

\subsection{Data collection}

This study was conducted by retrospectively reviewing CPR reports and medical records.

Medical records and CPR reports related to cardiac arrest were first reviewed primarily by the CPR Committee and subsequently by two emergency physicians following the AHA guidelines and the in-hospital utstein-style report template revised in 2020 .

\subsection{Variables}

Variables were classified into three categories: patient-related factors, pre-cardiac arrest factors, and CPR-related factors. Patient-related factors included age, sex, predisposing factors, requirement of vasopressor/mechanical ventilator during hospitalization, and causes of cardiac arrest. Pre-cardiac arrest factors included the medical department category at hospitalization, length of hospital stay, location of cardiac arrest, and presence or absence of monitoring before cardiac arrest. CPRrelated factors included time of event, location of arrest, initial witness, initial rhythm, defibrillation time, basic life support (BLS) activation time (first CPR time), ACLS activation time (CPR team arrival time), time to first epinephrine infusion, 
TA B L E 2. Features and interventions of cardiac arrest event by survival to discharge

Variables Survival to discharge $(n=132)$ In-hospital death $(n=473)$ Total $(n=605)$

Pre-arrest factors

Illness category, $\mathrm{n}(\%)$

Medical, cardiac

Medical, non-cardiac

$25(18.9)$

$61(46.2)$

9 (34.1)

$37(28.0)$

Surgical, non-cardiac

Trauma

$2(1.5)$

Length of hospital stay

(median, IQR)
ECG monitoring
Use of vasopressors

Mechanical ventilation

\section{Yes, n (\%)}

No, n (\%)

Yes, n (\%)

No, n $(\%)$

Yes, $\mathrm{n}(\%)$

No, n (\%)

CPR related factors and process

time of event

Day ( 7 am to $7 \mathrm{pm}$ )

Night or holiday

Location, n (\%)

ICU

Telemetry unit

Non-telemetry unit

Bystander, n (\%)

Doctor

Nurse

Healthcare worker

Other

$\begin{array}{ll}\text { Witnessed } & \text { Yes, n (\%) } \\ & \text { No, n (\%) }\end{array}$

Initial rhythm, n (\%)

$\mathrm{VF} / \mathrm{pVT}$

PEA

Asystole

Unknown

Defibrillation

$$
\text { Yes, n (\%) }
$$$$
\text { No, n (\%) }
$$

Defibrillation time, $\min$ (median, IQR, $\mathrm{n}=99)^{a}$

BLS activation time, min (median, IQR) ${ }^{b}$

ACLS activation time, min (median, IQR) $^{c}$

Time to first epinephrine, $\min$ (median, IQR) $^{d}$

CPR duration time, min (median, IQR)

\begin{tabular}{lcc} 
& Yes, n (\%) & $13(9.8)$ \\
ECPR & No, n (\%) & $119(90.2)$ \\
\hline
\end{tabular}

$104(78.8)$

28 (21.2)

$29(22.0)$

103 (78.0)

43 (32.6)

89 (67.4)

$49(37.1)$
$83(62.9)$
$63(47.7)$
$24(18.2)$
$45(34.1)$
$68(51.5)$
$43(32.6)$
$0(0)$
$21(15.9)$
$123(93.2)$
$9(6.8)$

$26(19.7)$

$72(54.5)$

15 (11.4)

19 (14.4)

32 (24.2)

100 (75.8)

$0.0(0.0-0.0)$

$0.0(0.0-1.0)$

$0.5(0.0-2.0)$

$2.0(0.0-5.0)$

$$
\begin{gathered}
54(11.4) \\
294(62.2) \\
11(2.3) \\
114(24.1) \\
27(0.4)
\end{gathered}
$$$$
3.0(3.0-10.5)
$$

$11.0(6.5-17.7)$

VF, Ventricular fibrillation;
9.0 (3.0-21.5)

323 (68.3)

$150(31.7)$

$169(35.7)$

$304(64.3)$

$144(30.4)$

$329(69.6)$

$170(35.9)$

303 (64.1)

$184(38.9)$

$110(23.3)$

$179(37.8)$

223 (47.2)

$178(37.6)$

7 (1.5)

65 (13.7)

$399(84.4)$

74 (15.6)

$52(11.0)$

$233(49.3)$

$74(15.6)$

$114(24.1)$

$67(14.2)$

406 (85.8)

$0.0(0.0-2.7)$

$0.0(0.0-1.0)$

$0.5(0.0-1.75)$

$3.0(1.0-5.0)$

26.5 (12.0-38.0)

$23(4.9)$
$450(95.1)$
79 (13.0)

355 (58.7)

$20(3.3)$

$151(25.0)$

29 (4.8)

$<0.01 *$

$8.0(3.0-21.0)$

$0.04 *$

427 (70.6)

178 (29.4)

198 (32.7)

407 (67.3)

$187(30.9)$

418 (69.1)

$0.01 *$

$<0.01 *$

0.63

219 (36.2)

0.80

$386(63.8)$

247 (40.8)

$134(22.1)$

0.16

$224(37.1)$

291 (48.1)

$221(36.5)$

7 (1.2)

86 (14.2)

$522(86.3)$

83 (13.7)

$<0.01 *$

78 (12.9)

305 (50.4)

87 (14.4)

$133(22.0)$

99 (16.4)

$506(83.6)$

0.33

$0.0(0.0-2.0)$

$<0.01 *$

0.66

$0.0(0.0-1.0)$

0.76

$0.0(0.0-2.0)$

0.95

0.43

$2.0(0.0-5.0)$

$<0.01 *$

$36(6.0)$

$569(94.0)$

$0.03 *$ time, First seen time to CPR team activation time or doctor arrival; ${ }^{d}$ Time to epinephrine: First seen time to first epinephrine injection time. 
TA B L E 3. Logistic regression model with survival to discharge

\begin{tabular}{|c|c|c|c|c|c|}
\hline & \multicolumn{2}{|l|}{ Univariate } & \multicolumn{2}{|l|}{ Multivariate } \\
\hline & & OR $(95 \% \mathrm{Cl})$ & $P$ value & OR $(95 \% \mathrm{Cl})$ & $P$ value \\
\hline \multirow{5}{*}{ Predisposing factors } & Sepsis & $0.30(0.16-0.55)$ & $<0.01^{*}$ & $0.37(0.20-0.66)$ & $<0.01 * *$ \\
\hline & ACS, this admission & $1.75(1.05-2.92)$ & $0.03 *$ & $1.77(0.89-3.54)$ & 0.10 \\
\hline & Malignancy & $0.43(0.26-0.70)$ & $<0.01 *$ & $0.37(0.20-0.66)$ & $<0.01 * *$ \\
\hline & Pneumonia & $0.40(0.23-0.70)$ & $<0.01 *$ & $0.44(0.23-0.83)$ & $0.01 * *$ \\
\hline & & \multicolumn{3}{|c|}{0.02} & 0.69 \\
\hline \multirow{6}{*}{ Causes } & Cardiac & \multicolumn{4}{|c|}{ Reference } \\
\hline & Respiratory & $0.74(0.45-1.22)$ & 0.24 & $1.21(0.61-2.42)$ & 0.57 \\
\hline & Septic & $0.27(0.12-0.58)$ & $<0.01 *$ & $0.56(0.20-1.53)$ & 0.26 \\
\hline & Hypovolemic & $0.64(0.31-1.31)$ & 0.22 & $0.87(0.36-2.10)$ & 0.76 \\
\hline & Other & $0.44(0.17-1.14)$ & 0.09 & $1.21(0.37-3.98)$ & 0.74 \\
\hline & Unknown & $0.63(0.33-1.21)$ & 0.16 & $0.94(0.41-2.15)$ & 0.89 \\
\hline \multirow{2}{*}{\multicolumn{2}{|c|}{ Length of hospital stay }} & $0.99(0.99-1.00)$ & 0.52 & $0.99(0.98-1.00)$ & 0.33 \\
\hline & & & \multicolumn{2}{|l|}{$<0.01 *$} & $0.01 * *$ \\
\hline \multirow{5}{*}{ Illness category } & Medical, non-cardiac & \multicolumn{4}{|c|}{ Reference } \\
\hline & Medical, cardiac & $2.23(1.28-3.86)$ & $<0.01 *$ & $0.90(0.44-1.86)$ & 0.79 \\
\hline & Surgical, non-cardiac & $1.93(1.20-3.13)$ & $<0.01 *$ & $1.91(1.08-3.37)$ & $0.02 * *$ \\
\hline & Surgical, cardiac & $3.94(1.56-9.92)$ & $<0.01 *$ & $2.23(0.73-6.80)$ & 0.15 \\
\hline & Trauma & $0.35(0.08-1.54)$ & 0.16 & $0.23(0.05-1.07)$ & 0.06 \\
\hline \multicolumn{2}{|l|}{ ECG monitoring } & $1.72(1.08-2.73)$ & $0.02 *$ & $1.16(0.63-2.13)$ & 0.62 \\
\hline \multicolumn{2}{|c|}{ Use of vasopressors, yes } & $0.50(0.32-0.79)$ & $<0.01^{*}$ & $0.28(0.16-0.50)$ & $<0.01 * *$ \\
\hline \multirow{2}{*}{\multicolumn{2}{|c|}{ Witnessed }} & $2.53(1.23-5.21)$ & $0.01 *$ & $1.46(0.63-3.35)$ & 0.37 \\
\hline & & & $<0.01 *$ & & 0.86 \\
\hline \multirow{4}{*}{ Initial rhythm } & $\mathrm{VF} / \mathrm{pVT}$ & \multicolumn{4}{|c|}{ Reference } \\
\hline & PEA & $0.61(0.36-1.06)$ & 0.08 & $1.54(0.54-4.36)$ & 0.41 \\
\hline & Asystole & $0.40(0.19-0.83)$ & $0.01 *$ & $1.44(0.43-4.86)$ & 0.54 \\
\hline & Unknown & $0.33(0.16-0.65)$ & $<0.01 *$ & $1.34(0.40-4.44)$ & 0.62 \\
\hline \multicolumn{2}{|l|}{ Defibrillation, yes } & $1.93(1.20-3.11)$ & $<0.01^{*}$ & $2.48(1.36-4.53)$ & $<0.01 * *$ \\
\hline \multicolumn{2}{|l|}{ ECPR, yes } & $2.13(1.05-4.34)$ & $0.03 *$ & $5.10(2.01-12.96)$ & $<0.01 * *$ \\
\hline \multicolumn{2}{|l|}{ CPR duration time } & $0.92(0.90-0.94)$ & $<0.01 *$ & $0.91(0.88-0.93)$ & $<0.01 * *$ \\
\hline
\end{tabular}

Nagelkerke's $R^{2}=0.363$, Hosmer \& Lemeshow: $P=0.19, x^{2}=11.15$

ACS: Acute coronary syndrome, VF: Ventricular fibrillation, $p V T$ : pulseless ventricular tachycardia, PEA:

Pulseless Electrical Activity, ECPR: Extracorporeal cardiopulmonary resuscitation.

\subsection{Statistics}

The collected data were analyzed using SPSS Statistics ver. 26.0 (IBM Corp., Armonk, NY, USA). Categorical variables (independent and dependent variables) were evaluated using the chi-square test. Continuous variables were tested for normality, and groups were compared using Student's $t$ test for parametric data and the Mann-Whitney $\mathrm{U}$ test for non-parametric data. Values of $P<0.05$ were considered statistically significant, with a $95 \%$ confidence interval (CI). Additionally, for survival to discharge rate, multivariate logistic regression analysis was conducted to compare and check the odds ratio (OR) and 95\% CI.

\section{Results}

\subsection{Baseline characteristics}

In total, there were 209,955 (average of 39,990 per year) hospitalized patients during the period, and 4,227 patients died during hospitalization, with an average of 805 deaths per year. During the study period, a total of 767 patients aged $\geq 18$ years received CPR for cardiac arrest during hospitalization. Of these, 14 patients developed cardiac arrest in the operating room, while 20 patients developed cardiac arrest during other procedures, including coronary angiography. There were 9 cases of cardiopulmonary resuscitation in situations where a DNR order had been previously written, and 51 cases were discontinued after the creation of a DNR order at the request 
TA B L E 4. Factors affecting CPC score in survival to discharge

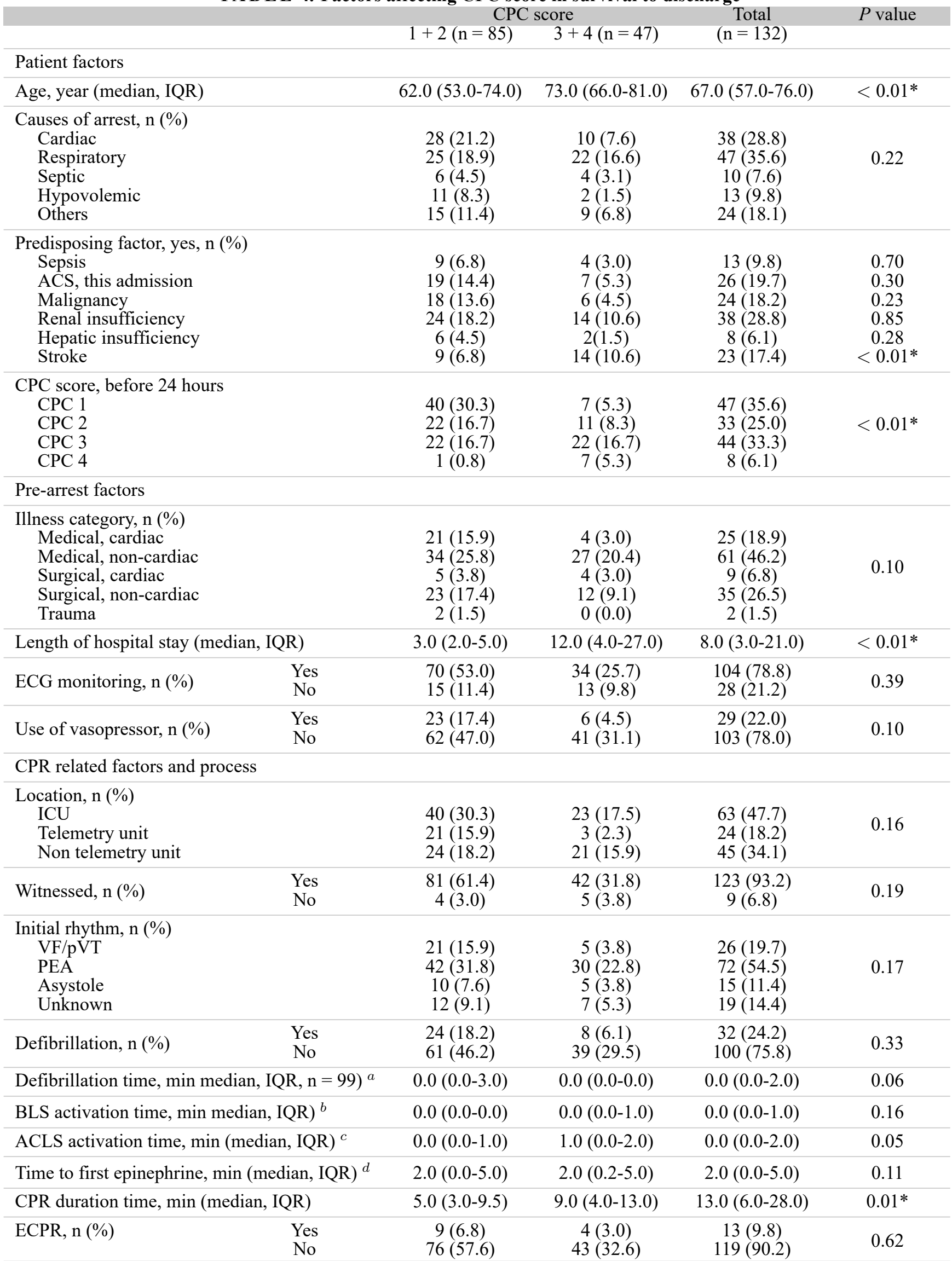

ACS: Acute coronary syndrome, VF: Ventricular fibrillation, $p V T$ : pulseless ventricular tachycardia, PEA: Pulseless

Electrical Activity, ECPR: Extracorporeal cardiopulmonary resuscitation.

${ }^{a}$ Defibrillation time: First recognized shockable rhythm time to defibrillation time, ${ }^{b}$ BLS activation time: First seen time to CPR start, ${ }^{c}$ ACLS activation time: First seen time to CPR team activation time or doctor arrival, ${ }^{d}$ Time to epinephrine:

First seen time to first epinephrine injection time. 


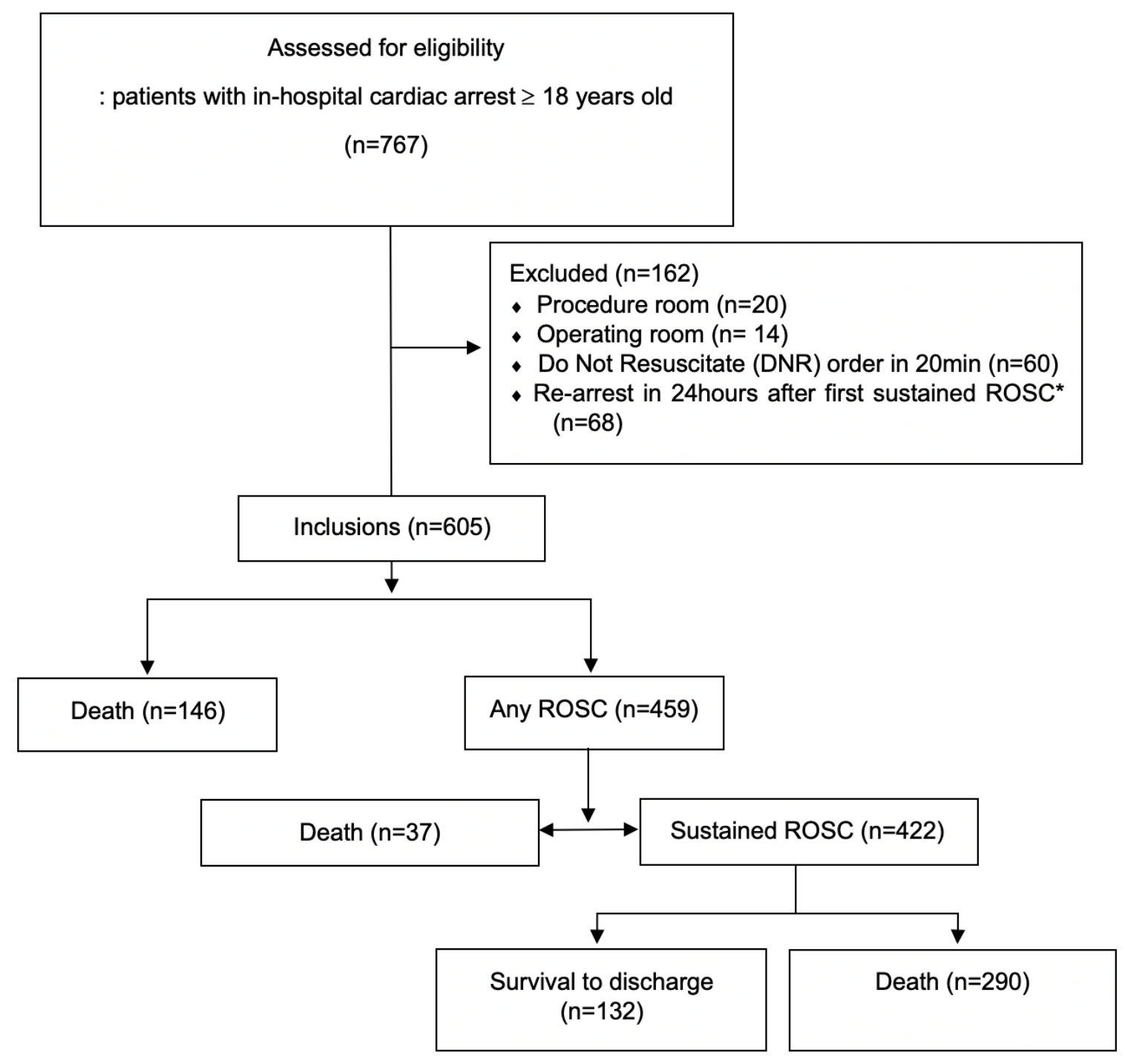

F I G U R E 1. Patients' flow diagram. ROSC*: Return of Spontaneous Circulation.

of a guardian within 20 minutes during CPR. Further, 68 cases of cardiac arrest occurred within 24 hours. The study was conducted on a total of 605 patients, excluding these 162 cases (Fig. 1).

Of the 605 patients, $69.7 \%(n=422)$ had their spontaneous circulation maintained for 20 minutes or more, and 36 of them maintained circulation with extracorporeal membrane oxygenation (ECMO).

The 24-hour, survival-to-discharge, and 1-month survival rates were $69.7 \%(422 / 605), 21.8 \%(132 / 605)$, and $20.3 \%$ (120/590), respectively. Good neurological prognoses (CPC $1,2)$ were achieved in $14 \%(85 / 605)$ of all patients with ROSC and $64.4 \%(85 / 132)$ of survivors who were eventually discharged.

\subsection{Comparison of the survival to discharge and in-hospital death groups}

The median age of the patients was 67 years; $61.6 \%(n=373)$ of the patients were men, and no statistical differences were observed between the survival to discharge and in-hospital death groups $(P=0.39, P=0.37)$. The causes of cardiac arrest and the presence or absence of certain comorbidities were included as patient factors affecting survival to discharge. The survival rate from cardiac arrest was better in cases with a cardiac cause (acute coronary syndrome, heart failure, arrhythmia, cardiac tamponade, etc.) than in those with a noncardiac cause $(P<0.01)$. Similarly, the survival rate was higher for patients hospitalized with acute coronary syndrome than for patients with other comorbidities $(P=0.03)$ (Table $3)$. The in-hospital death group significantly differed from the survival-to-discharge group with regard to presence of sepsis (9.4\% vs. $90.6 \%, P<0.01$ ), presence of ACS during the current admission $(31.0 \%$ vs. $69.0 \%, P=0.02)$, presence of malignancy $(13.0 \%$ vs. $87.0 \%, P<0.01)$, and presence of pneumonia $(11.9 \%$ vs. $88.1 \%, P<0.01)$ (Table 1$)$.

The classification of medical departments at the time of admission was a significant pre-cardiac arrest factor related to survival to discharge. When hospitalized for cardiac causes, both medical and surgical survival rates were high $(P<0.01)$ (Table 2).

Pre-cardiac arrest factors associated with survival to discharge included the use of vasopressors before cardiac arrest 
$(P<0.01)$, shorter length of hospital stay $(P=0.04)$, witnessed cardiac arrest, and electrocardiogram (ECG) monitoring at the time of discovery $(P=0.01$ and $P=0.02$, respectively). When the initial rhythm was ventricular fibrillation or ventricular tachycardia, the survival to discharge rate was $33.3 \%(26 / 78)$, which was statistically significant $(P<0.01)$. In addition, the survival to discharge rate was higher when defibrillation was performed, irrespective of the initial rhythm, than when defibrillation was not performed $(P<0.01)$. Extracorporeal cardiopulmonary resuscitation (E-CPR) was performed in 36 patients, of which $36.1 \%$ (13/36) were discharged alive $(P$ $=0.03$ ) (Table 2). The location of cardiac arrest and the first witness were not associated with survival to discharge. However, the intensive care unit tended to have a higher survival to discharge rate of $25.5 \%(63 / 247)$ than other locations; however, no statistical significance was observed.

The time from initial witness to CPR (referred to as BLS activation time) and CPR team activation time (referred to as ACLS activation time) were not significantly different within 1 minute in both groups $(P=0.76, P=0.95)$.

\subsection{Factors affecting survival to discharge}

Survival-to-discharge rate significantly decreased when hospitalization was accompanied by sepsis, pneumonia, or a history of malignancy ( $P<0.01, P=0.01, P<0.01$, respectively). On the chi-square test, the survival-to-discharge rate was observed to be higher in cases accompanied by acute coronary syndrome than in cases with other comorbidities; however, this was not significant in the multivariate analysis (OR 1.77, 95\% CI 0.89$3.54, P=0.10)$.

Multivariate analysis showed that the medical department at the time of cardiac arrest was significantly associated with the survival rate for non-cardiac surgery (OR: $1.91 ; 95 \% \mathrm{CI}$ : 1.08-3.37). The survival-to-discharge rate of patients with cardiac arrest in whom at least one defibrillation was performed during CPR, regardless of the initial rhythm, was 2.48 times higher (OR: 2.48; 95\% CI: 1.36-4.53). However, in the case of ventricular fibrillation or pulseless ventricular tachycardia, which is the initial rhythm requiring defibrillation, it was not significant when performing multivariate analysis with or without defibrillation. The survival rate was 5.1 times higher (OR: 5.1; 95\% CI: 2.01-12.96) in E-CPR than in standard CPR. A shorter CPR time corresponded with a higher survival rate (OR: 0.91, 95\% CI: 0.88-0.93).

In summary, factors directly related to the survival-todischarge rate included medical department at the time of admission; accompanying diseases, such as sepsis, malignant tumor, and pneumonia; use of vasopressors; defibrillation; E-CPR; and CPR duration time (Table 3).

\subsection{Factors affecting cerebral performance status and neurological prognosis}

The predictors of neurological outcomes are shown in Table 4. Young age, short length of hospital stay, and better neurological condition 24 hours before cardiac arrest were associated with better neurological outcomes $(\mathrm{CPC} \leq 2)$ (each $P<0.01)$.

Similarly, the presence of neurological disease at hospitalization was associated with a poorer neurological outcome $(P$
$<0.01)$.

For CPR-related factors, shorter CPR duration was associated with a better neurological outcome (each $P<0.01)$. However, no difference in BLS activation time, ACLS activation time, and defibrillation time was observed between CPC scores 1 and 2 and CPC scores 3 and 4 groups (Table 4 ).

\section{Discussion}

Despite active management and advanced resuscitation, IHCA is considered to have a poor survival rate and neurological outcome. The incidence of IHCA varies among countries and regions, ranging from $0-45 \%$; however, it is estimated to be approximately $20 \%$. In the United States, the quality of CPR has improved; indicators are continuously monitored, and CPR training is continuously conducted owing to the revision of CPR guidelines every 5 years based on scientific evidence [15].

According to the Get With The Guidelines-Resuscitation (GWTG-R) registry, the incidence of IHCA in the United States is estimated to be 292,000 cases per year, approximately 9-10 cases per 1000 people. Survival-to-discharge rates vary widely from 0 to $42 \%$; however, it is roughly $20 \%$, and it was estimated as $25 \%$ in 2017 [16, 17]. Of these, $85 \%$ were discharged with good neurological outcomes. Since the introduction of the GWTG-R system and the In-hospital Utstein style, the United States has been continuously managing the quality of IHCA patients, and the survival rate has improved over the past 20 years $[15,18,19]$.

In this study, patient-related factors significantly associated with survival to discharge included cause of cardiac arrest, underlying disease, and medical department at the time of admission. Survival rates were higher when the underlying cause of hospitalization was cardiac, especially for myocardial infarction or heart failure. This association may be due to the rapid action of ECMO insertion and defibrillation in cardiac arrest since patients with heart disease require close observation, including ECG monitoring. In the United States, according to Lars W. et al. and the GWTG-R registry in 2019, about 50$60 \%$ of cardiac arrest cases were due to cardiac causes $[15,16]$. In Korea, GR Chon et al. reported that non-cardiogenic causes were responsible for $66 \%$ of IHCA cases [20]. The $21 \%$ of IHCA cases caused by cardiac-related factors reported in this study is lower than that in other studies, which may be due to the exclusion of cardiac arrest cases that occurred during surgery or procedures, such as coronary angiography.

Although many patients suffer from internal diseases, and surgery is often required, cardiac causes are the most important factor causing cardiac arrest; however, other diseases often simultaneously induce cardiac arrest. Supposedly, most patients admitted to the hospital have various complex medical conditions, and IHCA may not be due to one disease. Therefore, in IHCA, classification and application of reversible causes of cardiac arrest [ $(5 \mathrm{H}$-hypoxia, hypovolemia, hydrogen ion, hypo/hyperkalemia, hypothermia) and (5T-toxins, tension pneumothorax, cardiac tamponade, coronary or pulmonary thrombosis, trauma)], as in OHCA, may help restore spontaneous circulation and improve survival and neurological outcomes [21].

The survival-to-discharge rate was significantly lower in 
patients with underlying diseases such as sepsis, pneumonia, and malignant tumors and when vasopressors were required. Several studies have reported that malignant tumors, sepsis, brain function status, pneumonia, hypotension, and underlying diseases, such as kidney and liver disease, can predict poor prognosis $[6,22,23]$. Another study reported that factors such as increasing age, male sex, active malignancy, and chronic kidney disease were associated with reduced survival rates [7]. Therefore, patients with these conditions should be identified as having a higher risk of cardiac arrest and closely observed.

In this study, approximately $60 \%$ of cardiac arrest cases occurred in the general wards. Although not statistically significant, the survival-to-discharge rate was high when cardiac arrest occurred in the intensive care unit. This finding is different from that observed for the GWTG-R system and others, where large-scale multicenter research was conducted. In these studies, cardiac arrest occurred more frequently in the intensive care unit in about $40 \%$ of total cases. In one study, the survival rate was highest in the telemetry unit in the general ward, followed by the intensive care unit and the non-telemetry unit in the general ward [24]. When a patient is hospitalized for severe disease or the disease condition is expected to worsen, patients are closely monitored. About $70 \%$ of patients with cardiac arrest in the hospital were monitored for the abovementioned reasons. The survival-to-discharge rate was higher with continuous ECG monitoring. This implies that there is a high survival-to-discharge rate even if cardiac arrest occurs when the patient's condition and disease progression are predicted in advance, and measures are taken to prevent cardiac arrest.

IHCA is expected in many cases because of existing diseases. Approximately $80 \%$ of patients reported that their condition had worsened 6 to 8 hours before cardiac arrest, and $70 \%$ of patients experienced worsening of symptoms because of respiratory problems 8 hours before cardiac arrest $[25,26]$. Approximately $10 \%$ of high-risk patients who could not be detected in the ward were reported to experience an unexpected cardiac arrest, accounting for $4 \%$ of all hospitalized patients [27]. It is possible to prevent more than $30 \%$ of unpredictable cardiac arrests by identifying high-risk patients in advance and continuously monitoring them [28].

Early recognition of cardiac arrest and activation of the CPR team, high-quality CPR, rapid defibrillation, appropriate advanced resuscitation, and post-cardiac arrest care are linked to the chain of survival and are currently considered essential elements of CPR. Several studies have reported "witnessed cardiac arrest" and cardiac arrest in about $80-85 \%$ and 50 $70 \%$ of patients already on an ECG monitor, respectively $[15,20,29,30]$. These measures are directly related to the patient's survival to discharge and prognosis. In this study, no statistical difference in CPR activation time was observed between the surviving and non-surviving groups. As described earlier, to improve the quality of CPR in the hospital where this study was conducted, our policy is to regulate and manage BLS and ACLS activation time within 3 minutes from the initial recognition of cardiac arrest. In addition, we intend to regularly monitor these indicators and conduct continuous quality control through CPR training. Since our code blue activation system is well managed within an average of 1 minute, there seems to be no difference between the surviving and nonsurviving groups, and the response time to code blue activation in general wards and intensive care units is considered to be similar.

According to some studies, only about $20 \%$ of the initial rhythms in cardiac arrest were shockable rhythm and required defibrillation; rapid defibrillation could lead to a 2-5 times or more increase in the survival rate $[6,31]$. In our study, the survival-to-discharge rate was 2.48 times higher $(95 \% \mathrm{CI}$ : 1.36-4.53, $P<0.01)$ when defibrillation was performed at least once, regardless of the initial rhythm, than when it was not performed. This highlights the importance of quickly identifying ventricular fibrillation or pulseless ventricular tachycardia, which are shockable rhythms, and providing defibrillation as soon as possible. Similar to the findings of an OHCA study [32], the survival rate increased in cases of witnessed arrest and initial shockable rhythm in our IHCA study.

In this study, the $64.4 \%$ of patients discharged with good neurological outcomes was significantly lower than the $85 \%$ reported in the US GWTG-R in 2017, which implies that the effects of integrated treatment, including targeted temperature management, cannot be overlooked [33, 34]. Younger age, shorter length of stay, witnessed cardiac arrest, and brain performance status with CPC scores of 1 and 2 were associated with good neurological prognosis. In another study, the survival-to-discharge rate increased when cardiac arrest occurred during daytime hours [6]; however, no statistical difference was observed in our study.

This study had several limitations. First, the study focused on a university hospital, which may not represent cardiac arrests in a general hospital and may introduce bias. Second, owing to its retrospective nature, missing and inaccurate records may have prevented the evaluation of other variables of interest. For example, the initial ECG records were often missing. In addition, the presence and monitoring of certain diseases were related to survival and prognosis. However, objective indicators (SOFA score, APACHE2 score) of disease severity at the time of hospitalization and cardiac arrest were not separately mentioned; thus, further investigation is necessary.

Finally, the 2020 AHA guidelines highlight the role of the rapid response team or the medical emergency team in cardiac arrest. In this study, the area of emphasis on survival to discharge and good neurological prognosis in patients with cardiac arrest was "continuous monitoring". However, in this study, the rapid response team mentioned earlier did not screen patients separately for early prevention and treatment of cardiac arrest; thus, a difference in the survival rate may be witnessed in other hospitals. In the future, multi-center research is required through sharing data and experiences between hospitals.

\section{Conclusions}

The compulsory availability of the code blue activation system is not sufficient. Patients at high risk of cardiac arrest should be identified, and a monitoring system should be established to identify abnormal signs and symptoms that can predict cardiac arrest. 


\section{AUTHOR CONTRIBUTIONS}

Ji Ho Ryu established the concept and planned the formal analysis and methodology, and reviewed and edited the writing. Byung Kwan Bae analyzed the data. Mun Ki Min, Dae Sup Lee, and Young Mo Cho validated the data. Soon Chang Park planned the methodology. Min Jee Lee wrote the original draft. Seok Ran Yeom and Mun Ki Min reviewed and edited the writing.

\section{ETHICS APPROVAL AND CONSENT TO PARTICIPATE}

This study protocol was reviewed and approved by the Institutional Review Board of our hospital (approval no. 05-2020163).

\section{ACKNOWLEDGMENT}

This research was supported by Pusan National University Research Grant, 2020. We would like to thank Editage (www . editage.co.kr) for English language editing.

\section{CONFLICT OF INTEREST}

The authors declare that there is no conflict of interest regarding the publication of this article.

\section{DATA AVAILABILITY}

The data used to support the findings of this study are available from the corresponding author upon request.

\section{REFERENCES}

[1] Merchant RM, Topjian AA, Panchal AR, Cheng A, Aziz K, Berg KM, et al. Part 1: executive summary: 2020 American Heart Association guidelines for cardiopulmonary resuscitation and emergency cardiovascular care. Circulation. 2020; 142: S337-S357.

[2] McEvoy MD, Field LC, Moore HE, Smalley JC, Nietert PJ, Scarbrough $\mathrm{SH}$. The effect of adherence to ACLS protocols on survival of event in the setting of in-hospital cardiac arrest. Resuscitation. 2014; 85: 82-87.

[3] Link MS, Berkow LC, Kudenchuk PJ, Halperin HR, Hess EP, Moitra VK, et al. Part 7: adult advanced cardiovascular life support. Circulation. 2015; 132: S444-S464.

[4] Kronick SL, Kurz MC, Lin S, Edelson DP, Berg RA, Billi JE, et al. Part 4: systems of care and continuous quality improvement: 2015 American Heart Association Guidelines Update for Cardiopulmonary Resuscitation and Emergency Cardiovascular Care. Circulation. 2015; 132: S397-S413.

[5] Kleinman ME, Brennan EE, Goldberger ZD, Swor RA, Terry M, Bobrow BJ, et al. Part 5: adult basic life support and cardiopulmonary resuscitation quality: 2015 American Heart Association Guidelines Update for Cardiopulmonary Resuscitation and Emergency Cardiovascular Care. Circulation. 2015; 132: S414-S435.

[6] Carrick RT, Park JG, McGinnes HL, Lundquist C, Brown KD, Janes WA, et al. Clinical predictive models of sudden cardiac arrest: a survey of the current science and analysis of model performances. Journal of the American Heart Association. 2020; 9: e017625.

[7] Fernando SM, Tran A, Cheng W, Rochwerg B, Taljaard M, Vaillancourt $\mathrm{C}$, et al. Pre-arrest and intra-arrest prognostic factors associated with survival after in-hospital cardiac arrest: systematic review and metaanalysis. British Medical Journal. 2019; 367: 16373.

[8] Sandroni C, Nolan J, Cavallaro F, Antonelli M. In-hospital cardiac arrest: incidence, prognosis and possible measures to improve survival. Intensive Care Medicine. 2007; 33: 237-245.

[9] Peberdy MA, Kaye W, Ornato JP, Larkin GL, Nadkarni V, Mancini ME, et al. Cardiopulmonary resuscitation of adults in the hospital: a report of 14720 cardiac arrests from the National Registry of Cardiopulmonary Resuscitation. Resuscitation. 2003; 58: 297-308.

[10] Hodgetts TJ, Kenward G, Vlackonikolis I, Payne S, Castle N, Crouch R, et al. Incidence, location and reasons for avoidable in-hospital cardiac arrest in a district general hospital. Resuscitation. 2002; 54: 115-123.

[11] Schein RMH, Hazday N, Pena M, Ruben BH, Sprung CL. Clinical antecedents to in-hospital cardiopulmonary arrest. Chest. 1990; 98: 13881392.

[12] Yoon H, Kwon Y, An J, Hong S, Kim YT. Main outcomes of the sudden cardiac arrest survey 2006 to 2016. Clinical and Experimental Emergency Medicine. 2019; 6: 183-188.

[13] Choi Y, Kwon IH, Jeong J, Chung J, Roh Y. Incidence of adult inhospital cardiac arrest using national representative patient sample in Korea. Healthcare Informatics Research. 2016; 22: 277.

[14] Nolan JP, Berg RA, Andersen LW, Bhanji F, Chan PS, Donnino MW, et al. Cardiac arrest and cardiopulmonary resuscitation outcome reports: update of the utstein resuscitation registry template for inhospital cardiac arrest: a consensus report from a task force of the International Liaison Committee on Resuscitation (American Heart Association, European Resuscitation Council, Australian and New Zealand Council on Resuscitation, Heart and Stroke Foundation of Canada, InterAmerican Heart Foundation, Resuscitation Council of Southern Africa, Resuscitation Council of Asia). Circulation. 2019; 140: e746-e 757

[15] Andersen LW, Holmberg MJ, Berg KM, Donnino MW, Granfeldt A. In-hospital cardiac arrest: a review. . Journal of the American Medical Association. 2019; 321: 1200-1210.

[16] Holmberg MJ, Ross CE, Fitzmaurice GM, Chan PS, Duval-Arnould J, Grossestreuer AV, et al. Annual incidence of adult and pediatric inhospital cardiac arrest in the United States. Circulation. Cardiovascular Quality and Outcomes. 2019; 12: e005580.

[17] Merchant RM, Yang L, Becker LB, Berg RA, Nadkarni V, Nichol G, et al. Incidence of treated cardiac arrest in hospitalized patients in the United States*. Critical Care Medicine. 2011; 39: 2401-2406.

[18] Benjamin EJ, Virani SS, Callaway CW, Chamberlain AM, Chang AR, Cheng S, et al. Heart disease and stroke statistics-2018 update: a report from the American Heart Association. Circulation. 2018; 137: e67-e492.

[19] Girotra S, Nallamothu BK, Spertus JA, Li Y, Krumholz HM, Chan PS. Trends in survival after in-hospital cardiac arrest. New England Journal of Medicine. 2012; 367: 1912-1920.

[20] Chon GR, Lee J, Shin Y, Huh JW, Lim C-, Koh Y, et al. Clinical outcomes of witnessed and monitored cases of in-hospital cardiac arrest in the general ward of a university hospital in Korea. Respiratory Care. 2013; 58: 1937-1944.

[21] Tirkkonen J, Hellevuo H, Olkkola KT, Hoppu S. Aetiology of in-hospital cardiac arrest on general wards. Resuscitation. 2016; 107: 19-24.

[22] Ebell MH, Afonso AM. Pre-arrest predictors of failure to survive after inhospital cardiopulmonary resuscitation: a meta-analysis. Family Practice. 2011; 28: 505-515.

[23] Chan PS, Spertus JA, Krumholz HM, Berg RA, Li Y, Sasson C, et al. A validated prediction tool for initial survivors of in-hospital cardiac arrest. Archives of Internal Medicine. 2012; 172: 947.

[24] Perman SM, Stanton E, Soar J, Berg RA, Donnino MW, Mikkelsen ME, et al. Location of in-hospital cardiac arrest in the united states-variability in event rate and outcomes. Journal of the American Heart Association. 2016; 5: e003638.

[25] Buist MD, Burton PR, Bernard SA, Waxman BP, Anderson J. Recognising clinical instability in hospital patients before cardiac arrest or unplanned admission to intensive care. A pilot study in a tertiary-care hospital. Medical Journal of Australia. 1999; 171: 22-25.

[26] Franklin C, Mathew J. Developing strategies to prevent inhospital cardiac arrest: analyzing responses of physicians and nurses in the hours before the event. Critical Care Medicine. 1994; 22: 244-247.

[27] Neale G, Woloshynowych M, Vincent C. Exploring the causes of adverse events in NHS hospital practice. Journal of the Royal Society of Medicine. 2001; 94: 322-330. 
[28] Lee BY, Hong S. Rapid response systems in Korea. Acute and Critical Care. 2019; 34: 108-116.

[29] Girotra S, Cram P, Spertus JA, Nallamothu BK, Li Y, Jones PG, et al. Hospital variation in survival trends for in-hospital cardiac arrest. Journal of the American Heart Association. 2014; 3: e000871.

[30] Brady WJ, Gurka KK, Mehring B, Peberdy MA, O'Connor RE. Inhospital cardiac arrest: impact of monitoring and witnessed event on patient survival and neurologic status at hospital discharge. Resuscitation. 2011; 82: 845-852.

[31] Chan PS, Krumholz HM, Nichol G, Nallamothu BK. Delayed time to defibrillation after in-hospital cardiac arrest. New England Journal of Medicine. 2008; 358: 9-17.

[32] Krishna CK, Showkat HI, Taktani M, Khatri V. Out of hospital cardiac arrest resuscitation outcome in North India -CARO study. World Journal of Emergency Medicine. 2017; 8: 200-205.

[33] Chan PS, Berg RA, Tang Y, Curtis LH, Spertus JA. Association between therapeutic hypothermia and survival after in-hospital cardiac arrest. Journal of the American Medical Association. 2016; 316: 1375.

[34] Chan PS, Nallamothu BK. Improving outcomes following in-hospital cardiac arrest: life after death. Journal of the American Medical Association. 2012; 307: 1917-1918.

How to cite this article: Min Jee Lee, Ji Ho Ryu, Mun Ki Min, Dae Sup Lee, Seok Ran Yeom, Byung kwan Bae, et al. Predictors of survival and good neurological outcomes after in-hospital cardiac arrest. Signa Vitae. 2021;17(2):67-76. doi:10.22514/sv.2021.009. 